Fecha de recepción: marzo 2020 Fecha de aceptación: abril 2020 Versión final: mayo 2020

\section{Diseño de materiales: del Basic design al Material Driven Design}

Jimena Alarcón ${ }^{(1)}$, Flaviano Celaschi ${ }^{(2)}$

y Manuela Celi ${ }^{(3)}$

Resumen: Este artículo aporta una visión respecto del diseño de materiales como temática del diseño industrial, generado desde la influencia proveniente del referente italiano basado en la filosofía proyectual de Carmelo Di Bartolo. Su objetivo es evidenciar las contribuciones del basic design vinculado a la biónica, aplicado a la construcción de un planteamiento metodológico proyectual del diseño industrial observante de los materiales que configuran los artificios. El basic design es el método basado en la observación de la naturaleza, a partir del cual los diseñadores extrapolan principios biónicos hacia la concepción del mundo artificial. La relevancia es la capacidad de integrar aportes al ámbito del ahorro energético, del material y un compromiso intrínseco con el medioambiente. No por contar con amplios recursos económicos, productivos y tecnológicos, el proceso creativo ha de ser ilimitado y excesivo. Se plantean aproximaciones que quedan expresadas en el proceso proyectual integrador denominado Material Driven Design, que incorpora el concepto de calidad percibida para integrar aspectos funcionales y emocionales de los materiales. Las conclusiones están referidas a las consideraciones que los tres aspectos (Basic design, biónica y Material Driven Design) aportan al proceso proyectual.

Palabras clave: biónica - calidad percibida - diseño industrial - metodología - sensorialidad.

[Resúmenes en inglés y portugués en la página 69]

(1) Doctora en Gestión del Diseño, fundadora y directora del Centro de Emprendimiento e Innovación en Diseño (2013). Ha sido profesora e investigadora del Departamento de Arte y Tecnologías del Diseño de la Universidad del Bío-Bío desde 1995.

(2) Doctor en Investigación, catedrático de Diseño en la Facultad de Arquitectura de la Universidad de Bologna, Italia, donde fundó el año 2012 el AdvancedDesign Network. Ha sido profesor catedrático de Diseño el Politécnico de Milán, Politécnico de Torino, entre otros.

(3) Doctora en Investigación en Diseño Industrial, investigadora del Departamento de Diseño del Politécnico de Milán. Desde 2011 es coordinadora del Humanities Design Lab. Ha profundizado las relaciones entre el diseño y el proyecto orientado al futuro. 


\section{Introducción}

Este artículo pone en valor un planteamiento basado en la observación de la naturaleza como referente para entregar respuestas proyectuales con fuerte base en la curiosidad, como motor fundacional de la innovación. La naturaleza demuestra ser una rica fuente de inspiración para el diseño de productos a lo largo de toda la historia (Emami et al., 2008). Sin duda, cuando se observa, se busca develar e interpretar, entre otros principios, el de la eficiencia, donde la biónica puede dar importante ayuda para generar ideas y soluciones integrales en el recorrido creativo del proyecto. Paralelamente, podemos entender a la biónica como "la asimilación de principios de ingeniería que se utilizan en sistemas naturales, y la aplicación de éstos al diseño o mejora de sistemas tecnológicos o materiales" (Lodato, 2000). Se subraya el reconocimiento al estudio de los sistemas naturales y mediante un proceso de abstracción, pueden enunciarse principios aplicables a la ingeniería, transferibles, a su vez, al ámbito de lo artificial (Miralles y Giuliano, 2008).En este sentido, según Di Bartolo (Di Bartolo et al., 2000), naturaleza, estructura y mínimo esfuerzo, son tres ingredientes relevantes a asociar en las prácticas de diseño experimental-conceptual con la biónica, considerando un análisis horizontal o de un modelo sistémico del patrimonio natural, observando la interdependencia de formas y soluciones entre ellas y con el ambiente; y, otro vertical, a partir del análisis de un modelo de la naturaleza referenciando las cualidades funcionales de las estructuras vegetales y animales, para codificar soluciones específicas del diseño de objetos cotidianos.La biónica estudia y construye sistemas artificiales por analogía con los vivos, evita las sugerencias formales y apunta a un doble movimiento, la observación y la recogida de datos en el momento de la investigación; y, la aplicación innovadora de aspectos formales, funcionales y estructurales, al momento del proyecto (Di Bartolo, 2000).La perspectiva general sitúa a la biónica como una metodología capaz de impulsar sistemáticamente el proceso de diseño. Observando y analizando la naturaleza, es posible comprender sus relaciones estructurales, organizativas, funcionales, expresivas y económicas, además de aplicar métodos que sistematizan estas relaciones analógicas (Alarcón, 2003).

Según Di Bartolo (2017), pensar con límite estimula la creatividad en la disciplina del diseño. Si no existe el límite, todo es autorreferencial. En esta medida, los estudiantes de primer año deben establecer desarrollos para superar este aspecto con el mínimo uso de energía. El diseñador tiene que responder a vínculos de usabilidad, ergonomía, materiales, mercado, experiencias, en síntesis, tiene que escuchar; en cambio el artista, no tiene que justificar nada a nadie. Hay una cantidad enorme de experiencias en la arquitectura, como por ejemplo la obra de Pier Luigi Nervi, Felix Candela y Buckminster Fuller, que trabajan para construir una vivienda, un edificio con el menor empleo de materiales, el mismo Gaudí que trabajaba con la catenaria, donde la curva se usa para identificar el menor recorrido de un punto a otro. Entonces, cuando se crea una experiencia a través de un desafío, se pueden idear trabajos y estructuras hiperestáticas, es decir, en equilibrio.

Di Bartolo desarrolla y aplica el método del basic design en entornos investigativos y educativos, dándose cuenta que los resultados constructivos geométricos de diseño son muy similares a la experiencia de la naturaleza. A partir de entonces, observa cómo la natura- 
leza soluciona un problema con una geometría apropiada, un mínimo gasto energético y uso de materiales, cumpliendo su objetivo eficientemente. Comienza a descubrir la experiencia con la biónica, que nace a partir de ese paso entre el basic design y su aplicación en el campo de los proyectos. Para Di Bartolo: "Uno de los temas de trabajo históricos del diseño es el deseo de garantizar al ser humano un entorno artificial armonioso; la biónica intenta hacerlo a través del modo de plantear problemas antes de las soluciones, [ella] se encuentra en particular sintonía con otra tendencia actual del proyecto: aquella que garantiza el máximo rendimiento de los objetos artificiales con el mínimo uso de energía. La biónica, con sus observaciones sobre refinadas soluciones compuestas que tienen éxito en el cuerpo de los animales y en la estructura de las plantas, puede formular preguntas más precisas e inéditas y dar un mejor uso a las tecnologías. Las características de la investigación biónica responden finalmente a tres de las necesidades más importantes del diseño contemporáneo: integración profunda de las soluciones funcionales y formales, el dinamismo en la adhesión a las necesidades del proyecto, la flexibilidad en la propuesta de soluciones. Por esta razón, la investigación biónica aparece como una herramienta efectiva disponible para el diseño contemporáneo (Di Bartolo, 1999). “El diseñador biónico aplica constantemente, hacia la naturaleza, un tipo de reverse engineering. Busca desvelar mecanismos funcionales que puedan ser útiles para sus objetos. Una vez que estos mecanismos se identifican, la operación de traspaso del dominio natural al artificial es una especie de re-interpretación de la naturaleza que funda la relación de semejanza" (Di Bartolo y Montanari, 2004, p.87). Para Di Bartolo, la biónica surge como un valor en su perspectiva proyectual, de la mano del basic design practicado con sus estudiantes en los inicios de su vida profesional, motivado por sus profesores Roberto Lucci y Paolo Orlandini, quienes a su vez trabajaban con los maestros del diseño de los años '60 y '70 (Zanusso, Castiglione, entre otros) y habían estudiado en el Illinois Institute of Technology en Chicago, en la Escuela de Mies van der Rohe, realizando esta tipología de ejercicio. El objetivo es comprender empíricamente fenómenos estructurales y formales, en busca de descubrir mecanismos o principios naturales para implementarlos en el mundo artificial. Todo lo anterior, en armónico complemento con lo expresado por Munari(1990), que señala que se toma como punto de partida un fenómeno natural y a partir de ahí se puede desarrollar una solución proyectual. De este modo, el basic design propicia una actitud de curiosidad, de búsqueda necesaria, ya que un diseñador que descubre, que desestructura, es capaz de crear. Realiza un aprendizaje del diseño basado en experiencias, en jugar, porque así se puede descubrir un mundo que antes no se pensaba. Ahora bien, tan importante como esta actitud, es crear una dinámica y una experiencia comunitaria, para crear una inteligencia colectiva en que todos disfrutan.

El diseño de materiales valora al proyecto del material como una práctica independiente a la concepción del producto final (Rognoli y Levi, 2005).En este sentido, el diseño del material, es anterior al diseño del producto, dado que los materiales pueden generarse para diversas prestaciones. Ejemplos en la historia del diseño, exploran lúdicamente diversas texturas y acabados, además de aspectos fenomenológicos sobre las ventajas (o desventajas) de utilizar materiales particulares para productos particulares (Karana et al., 2015). 
En este campo, un futuro más respetuoso y responsable con el medioambiente, exige la incorporación de múltiples variables, para evidenciar el potencial que los materiales tienen, abarca, por ejemplo, enfoques sustentables, inteligentes, como motor de impulso para el diseño y las sociedades (Ayala et al., 2011; Parisi et al., 2017; Camere y Karana, 2017; Karana et al., 2016).

En el contexto actual, tienen cabida reflexiones propias de la dimensión del significado, el vínculo del material con aspectos sensoriales, referidos a la calidad como objetivo para distinguir el producto de sus competidores. Se subraya el hecho que no se pueden ignorar dos consideraciones básicas: primero, percibimos lo que sabemos; y, en segundo lugar, la percepción está indisolublemente vinculada a los materiales (Design Innovation and Indesit Company, 2012).Una mirada contemporánea del proyecto en su fase conceptual, valora la potencialidad de la experiencia sensorial posible de percibir, a través de la expresión de los materiales, del significado que tienen por sí mismos. Tradicionalmente, el material es concebido como un operador elemental, cuya tarea principal consiste en dar estructura a un sistema más complejo; sin embargo, en la nueva situación, el material puede presentar una complejidad intrínseca, gestionada por el proyectista para dar presencia al objeto que lo hace capaz de realizar funciones (Manzini, 1986). Desde la perspectiva del diseño de materiales existen dos desarrollos posibles: el primero, está referido a las cualidades superficiales, que son las relativas al espectro visivo y formal en que se definen sus características expresivas y sensoriales (Lefteri, 2007), valorando la semántica del producto y la estética del material lograda mediante acabados; y, el segundo, que dice relación con las cualidades físico-mecánicas, que son las que definen las propiedades estructurales y prestaciones funcionales de un material, no siempre ligadas a su definición estética. "Los materiales forman parte del alma de un diseño y su belleza física y mental (estética) debe respetarse, apreciarse y disfrutarse (ética). Los materiales deben llevarse hasta límites inimaginables (desarrollo). Hay que dejarse cautivar por los materiales" (Bramston, 2010, p.80).

\section{La nueva filosofía del diseño de materiales a partir de Carmelo Di Bartolo}

La innovación de materiales para la industria no constituye una materia para los diseñadores hasta el advenimiento de los materiales poliméricos, es decir, hasta la introducción de la innovación de materias plásticas por parte del italiano Giulio Natta, premio Nobel en 1962. Hasta entonces, la innovación de materiales había estado indiscutiblemente en el territorio de la química y la ingeniería. A partir de los años 60, en Europa, pero más aún en los Estados Unidos de Norteamérica, los arquitectos y diseñadores valoran la idea de que el material puede ser diseñado. Una revolución que tiende a moverse en bloque hacia la cuestión del material innovador, es decir, de un discurso basado en las prestaciones hacia uno basado en la estética y la capacidad comunicativa del material. Con la innovación de la plástica y el color, el diseño, la alquimia, se traslada de ser un simple tratamiento superficial y de acabado con pintura de post producción, a ser pensado dentro de la concepción del producto integral: todos los materiales se colorean y vuelven maleables a voluntad. Transversalmente, podemos decir que la segunda revolución industrial trajo consigo la 
siempre muy visible y masiva posibilidad de recubrir al material artificial: primero, acero y aleaciones de metal, pasando por polímeros y materiales de diseño con tratamientos de ennoblecimiento y coloración superficial, tendiendo a ocultar siempre más y más las características tradicionales del material considerado natural, como ocurre en variadas ocasiones con la madera y la cerámica. El diseñador se apodera del diseño del producto relacionándolo con materiales artificiales, dejando a artesanos, ebanistas, ceramistas y maestros del vidrio, la sensibilidad y la capacidad de trabajar con materiales en estados más naturales.

En este contexto se inserta el trabajo -filosófico- de Carmelo Di Bartolo, sobre la nueva cultura del material que se apodera del estudio de la biónica, a través de las características que la naturaleza dota a plantas, flores y organismos vivientes, con el fin de referenciar el modo aprendido en orden de interpretarlo y perfeccionarlo en la práctica usada para la generación de innovación en diseño de materiales. Di Bartolo se pone al centro del debate entre lo natural y lo artificial, entre las razones estéticas y las prestacionales y prueba una inédita alianza para concebir el producto. Si bien el resultado artificial posee sus propias características, en este caso es también evidencia de una interpretación del modelo natural, sin renunciar a la forma y sus reacciones autónomas, permitiendo incluso mejorar la intención identitaria de la naturaleza. Podemos llamar estructuralista al esfuerzo de Di Bartolo, debido al desciframiento -hermenéutico- de la manifestación del sujeto natural y de la forma en que éste se expresa, ya que tiende a realizar un modelo no matemático de la naturaleza, con predominancia de la modelación biomimética en que lo estético y lo estructural se funden. El estudio de Di Bartolo sobre las formas naturales (Figura 1), es un referente de cómo el análisis y la interpretación acompañada de la mano del ser humano, puede convertirse en un producto con el valor propio de lo realizable industrialmente (Figura 2).

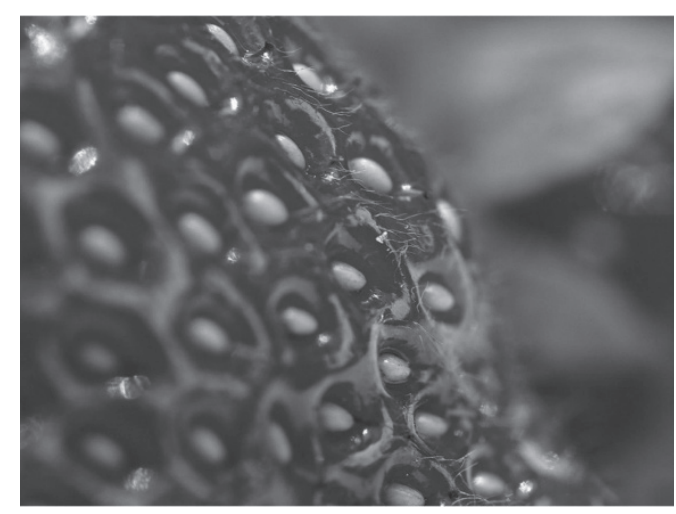

Figura 1: Referente biónico para diseñar texturas de material. Fuente: Carmelo Di Bartolo. 


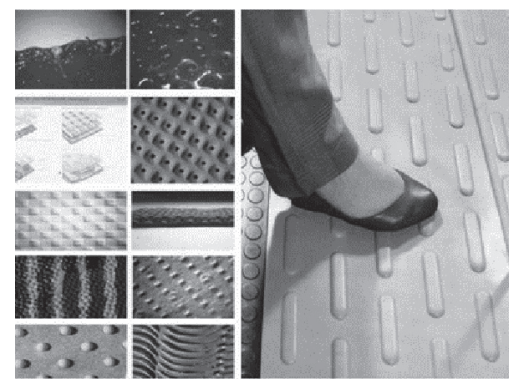

Figura 2: Material diseñado para Metropolitana de Milano. Fuente: Carmelo Di Bartolo.

\section{El enfoque Material Driven Design aplicado al diseño de materiales: el caso Eldomat}

Se trata de un proceso proyectual complejo y articulado que incorpora un modelo en que el diseño del material, influye sobre la realización de un producto de mercado, poniendo al centro de la investigación el tema de la percepción de la calidad del material, la dimensión del significado, el enlace con el aspecto táctil y sensorial, así como elemento diferenciador del producto respecto de la competencia. Su objetivo principal es generar y experimentar una metodología para caracterizar el material. La parte sustancial del caso Eldomat(Indesit Company \& Design Innovation, 2012), financiado por el Ministerio de Desarrollo de Italia, nace de la colaboración sinérgica de Indesit Company, (actual grupo Whirpool) y Carmelo Di Bartolo, desarrollando un trabajo que ha contribuido a un proceso de investigación complejo y articulado. Este caso será empleado para ejemplificar el rol estratégico del enfoque Materials Driven Design, el cual se aborda desde dos perspectivas: primero, la estrategia industrial orientada al mercado y a la dimensión sistémica; segundo, la investigación conectada a la calidad percibida y la selección del material. Para profundizar en estos aspectos tenemos:

1. Estrategia industrial orientada al mercado: se plantea a través de las fases de diseño y de la apropiada elección de materiales con potencial para creardistintos niveles de innovación, según los recursos disponibles y el valor que se desea promover. Eldomat desarrolla un proceso metodológico en cascada (Figura 3), que se inicia con la etapa 1, que considera como eje de estudio las marcas (Hotpoint Indesit y Scholtès), para definir la tipología de materiales que se aplicarán en los productos, incorporando una mirada estratégica basada en la investigación de mercado que involucra la definición de tendencias y oportunidades, generando nuevos inputs para la ideación de productos. La fase (a) Estrategia de Materiales permite evaluar y definir las valoraciones sensoriales y técnicas en consideración a sus características y futura integración en los productos finales. La etapa 2, se refiere a los 
grupos individuales (cocción, refrigeración y lavado), con el objetivo de generar conceptos innovadores que se definen, en la fase (b) como ideas materiales. Los resultados de la fase previa se filtran aquí, pasando de una selección estratégica a una contextualización para macro áreas denominados clústeres. En la etapa 3, se implementa la transferencia de las ideas materiales al producto final. Los requisitos para cada función del producto están determinados manteniendo una coherencia general con respecto a las marcas de referencia. Hablamos así del diseño de materiales, fase (c) que proviene de la combinación entre conceptos innovadores y aplicaciones de materiales inesperados.

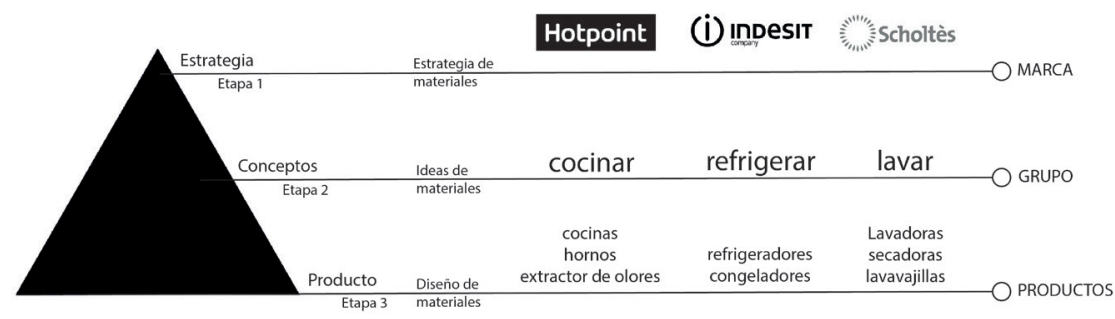

Figura 3: Enfoque como Materials Driven Design, Eldomat.

Fuente: Archivo proyecto basado en Eldomat.

2. Calidad percibida: tiene como objetivo ir más allá de los cinco sentidos y ahondar profundamente en las expectativas del usuario, tratando de objetivar la calidad del producto, a través de una selección de materiales capaces de transmitir consistencia con tales perspectivas. Considera tres aspectos para la caracterización del material; iniciando con la jerarquía sensorial como primer rasgo a considerar para el diseño, que va desde la primera apreciación e impacto visual, al conocimiento e interacción con él, permitiendo dar una valoración a la experiencia resultante de su uso repetido. Esta primera impresión es esencialmente visual, con atención en la capacidad de cada objeto para atraer la atención positivamente o causar rotundo rechazo. El siguiente paso es el conocimiento, que se realiza a través de una interacción táctil, acústica u olfativa del producto, permitiendo comprobar su desempeño y observancia de las leyes de la ergonomía física y epistémica, y; la experiencia, como resultado conclusivo, que integra en la percepción, a todos los sentidos, dominando la vista, pues se advierte que la visión contiene una amplia posibilidad de sensaciones, entre ellas, táctiles, acústicas y olfativas. El último aspecto a considerar es la evaluación perceptiva, resultante de la interacción con el usuario, la que puede ser de aceptación o desaprobación y, es determinante en la decisión de compra, impulsando al usuario a que se incline por la marca o producto que mejor desempeña sus promesas preliminares. 
El procedimiento implementado detecta las características sensoriales de los materiales y permite seleccionar aquellas coherentes con las características técnicas relacionadas con la función y el desempeño. Para la caracterización técnica se estudian las prestaciones, entre ellas: resistencia mecánica, ligereza, seguridad, performance, dureza, resistencia a la abrasión y ecología, mientras que para las características sensoriales se revisan aspectos propios de lo perceptivo, como, por ejemplo: sentido de limpieza, durabilidad, cuidado, confortabilidad, sentido ético, todos ellos aspectos que el producto comunica al usuario final, a través de su aspecto (Figura 4). La selección de los materiales efectuada a través de la metodología de percepción sensorial, permite generar una serie de conceptos innovadores basados en la tendencia de materiales que influencia las características estético-sensoriales de las prestaciones del producto, donde los atributos de los materiales son fundamentales para construir, en la primera impresión, una apreciación positiva.

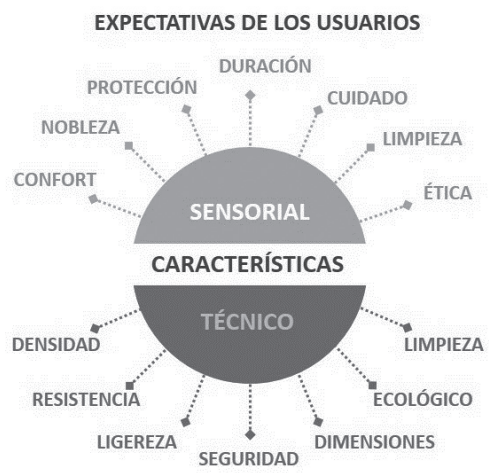

Figura 4: Características sensoriales y técnicas de los materiales. Fuente: Archivo proyecto basado en Eldomat.

La particularidad del enfoque formulado está en la capacidad de gestionar una conciencia proyectual caracterizada por un conocimiento visual, pero también en la habilidad para manipular la nueva complejidad del producto, su cualidad y el modo en que se expresa y percibe.

"Las comunidades creativas, los científicos y las industrias de materiales se están involucrando profundamente en el desafío creativo para lograr la funcionalidad y el significado de los materiales" (Caisse y Montreuil 2014, p.10), por lo que integrar estos aspectos en el proceso proyectual aporta una nueva dimensión del diseño. 


\section{Conclusiones}

La propuesta filosófica de Di Bartolo originada en el Basic design y la biónica y que decanta en el enfoque Material Driven Design, genera cambios en la cultura del proyecto, ya que implica pensar el artificio desde lo esencial que resulta el material constitutivo, elemento sugerente y comunicador, comprometido con la percepción de los usuarios. El basic design y la biónica, en su conjunto, permiten la realización de estudios empíricos, fórmulas proyectuales, procedimientos, técnicas y experiencias concretas para configurar materiales y objetos. Son herramientas diferenciadoras que permiten articularse al paradigma de sostenibilidad, debido a que cuando un material nace considerando el basic design y la bióni$\mathrm{ca}$, necesariamente integra conceptos de justeza y eficiencia asociados al ahorro de las materias que lo componen, así como de los procesos productivos y logísticos necesarios para su producción y distribución, respectivamente. La articulación con variables del enfoque Material Driven Design permite pensar la experiencia del usuario con el material diseñado, satisfaciendo requerimientos funcionales y sensoriales. Genera una ocasión para que la persona pueda tener una vivencia más confortable en esta interrelación. Bartolo define y traspasa una estrategia para concebir la idea de proyecto con la aplicación innovadora de aspectos formales, funcionales y estructurales al momento de la ideación. Derivado de esto, se incorpora en el proceso de diseño la dimensión de la interacción sensorial, innovativa y la sintonía con las necesidades funcionales y sensoriales de los usuarios, envueltas en el enfoque Material DrivenDesign.

\section{Agradecimientos}

Los autores agradecen a proyecto CONICYT No REDI170581 "Valor percibido de materias primas recicladas, aplicadas a la generación de nuevos materiales: diseño para la aceptación social y economía circular" (2017/2020).A Andrea Llorens Vargas, por su colaboración como ayudante de investigación para académicos, financiado por la Dirección de Investigación de la mencionada instituciónde la Universidad del Bío-Bío.

\section{Referencias}

Alarcón J. (2003). Biónica y Diseño Innovativo: el sector maderero y oportunidades para la generación de nuevos materiales. Tesis magister guiada por Carmelo Di Bartolo, Concepción, Chile.

Ayala C.; Quijano, A.; Ruge, C. (2011). Los materiales como medio para estimular procesos de creación. Dearq, Revista de Arquitectura 8, p.p. 44-53.https://doi.org/10.18389/ dearq8.2011.06

Bramston, D. (2010). Bases del diseño de producto 01: de la idea al producto. Barcelona: Parramón. vol. 2, p. 80. 
Caisse, S.; Montreuil, B. (2014). Polar Business Design. SAGE Open,January-March 2014, p.p. 1-16, p.10. https://doi.org/10.1177/2158244014522632

Camere, S.; Karana, E. (2017). Growing Materials for Product Design. In Alive. Active. Adaptive. Proceedings of International Conference on Experiential Knowledge and Emerging Materials (EKSIG June 19-20). Delft, the Netherlands, p.p. 101-115.

Di Bartolo, Carmelo. (1999). Bionica: lo sviluppo naturale nel progetto. Domus $n^{\circ} 818$.

Di Bartolo, C. (2000). Naturaleza como modelo, naturaleza como sistema. Experimenta Revista de Diseño y Comunicación para la Empresa, $n^{\circ} 31$, p.p. 9-45.

Di Bartolo, C.; Hennicke, J.; Nachtigall, W.; Plasencia, C.; Songel, G. (2000). La naturaleza como fuente de innovación. Valencia: Editorial UPV.

Di Bartolo, C.; Montanari, R. (2004). Complejidad, diseño y sociedad. Cuadernos de Diseño Pensar/Proyectar el Futuro. Madrid: Instituto Europeo de Diseño n ${ }^{\circ}$, p.87.

Di Bartolo, C. (2017). “Una vida dedicada al Diseño." Entrevistado por Jimena Alarcón en Design Innovation, Milán, Italia. Documento no publicado.

Design Innovation and Indesit Company. (2012). Materials driven design, Il progetto Eldomat. Design Fausto Lupetti (by Dodici Edizioni), Milano, 5-7.

Emami, J.; Tashakori, M.; Tashakorinia, Z. (2008). Bionic design in industrial design education at University of Tehran. Proceedings of E\&PDE, 10th International Conference on Engineering and Product Design Education.U. P. C., Barcelona, Spain, p.p. 435-440.

Karana E.; Barati, B.; Rognoli, V.; Zeeuw van der Laan, A. (2015). Material Driven Design (MDD): A method to design for material experiences. International Journal of Design 19, $\mathrm{n}^{\circ}$ 2, p.p. 35-54.http://resolver.tudelft.nl/uuid:7359026d-57f5-4f63-9835-126c5d23baed

Karana, E.; Pedgley, O.; Rognoli, V.; Korsunsky, A. (2016). Emerging Material Experiences. The Journal of Materials and Design vol. 90, p.p. 1248-1250. https://doi.org/10.1016/j. matdes.2015.07.042

Manzini, E. (1986). La materia dell'invenzione. Milano: Arcadia edizioni.

Miralles, M.; Giuliano, G. (2008). Biónica: eficacia versus eficiencia en la tecnología natural y artificial. Scientiae Studia, 6 (3), p.p. 359-369.http://dx.doi.org/10.1590/S167831662008000300005

Munari, B. (1990). ¿Cómo nacen los objetos? Barcelona: Gustavo Gili.

Lefteri, C. (2007). Materials for Inspirational Design. RotoVision. East Sussex, UK.

Lodato, F. (2000). Biónica: la naturaleza como herramienta de innovación. Experimenta, Revista de Diseño y Comunicación para la Empresa, $n^{\circ} 31$.

Parisi, P.; Rognoli, V.; Sonneveld, M. (2017). Material Tinkering. An inspirational approach for experiential learning and envisioning in product design education. The Design Journal,20: sup1. https://doi.org/10.1080/14606925.2017.1353059

Rognoli, V.; Levi, M. (2005) Materiali per il design: espressività e sensorialità. Milano: Polipress. 


\begin{abstract}
This paper provides a vision regarding the design of materials as a theme of industrial design, generated from the influence of the Italian referent based on the design philosophy of Carmelo Di Bartolo. Its objective is to demonstrate the contributions of basic design linked to bionics, applied to the construction of a projective methodological approach of the observant industrial design of the materials that make up the artifices. Basic design is the method based on the observation of nature, from which designers extrapolate bionic principles towards the conception of the artificial world. The relevance is the ability to integrate contributions to the field of energy savings, material and an intrinsic commitment to the environment. Not because of having extensive economic, productive and technological resources, the creative process must be unlimited and excessive. Approaches are presented that are expressed in the integrative project process called Material Driven Design, which incorporates the concept of perceived quality to integrate functional and emotional aspects of materials. The conclusions are related to the considerations that the three aspects (Basic design, bionics, and Material Driven Design) contribute to the design process.
\end{abstract}

Keywords: bionics - perceived quality - industrial design - methodology - sensoriality.

Resumo: Este artigo fornece uma visão sobre o design de materiais como um tema do design industrial, gerado a partir da influência do referente italiano baseado na filosofia de design de Carmelo Di Bartolo. Seu objetivo é demonstrar as contribuições do design básico ligado à biônica, aplicadas à construção de uma abordagem metodológica projetiva do design industrial observante dos materiais que compõem os artifícios. O design básico é o método baseado na observação da natureza, da qual os projetistas extrapolam princípios biônicos para a concepção do mundo artificial. A relevância é a capacidade de integrar contribuições ao campo de economia de energia, material e um compromisso intrínseco com o meio ambiente. Não por ter amplos recursos econômicos, produtivos e tecnológicos, o processo criativo deve ser ilimitado e excessivo. Abordagens são apresentadas que são expressas no processo de projeto integrativo chamado Material Driven Design, que incorpora o conceito de qualidade percebida para integrar os aspectos funcionais e emocionais dos materiais. As conclusões estão relacionadas às considerações que os três aspectos (design básico, biônica e design orientado a material) contribuem para o processo de design.

Palavras chave: biônica - qualidade percebida - desenho industrial - metodologia sensorialidade.

[Las traducciones de los abstracts fueron supervisadas por el autor de cada artículo] 